\title{
Rapid Assessment on Potential Risks of Schistosomiasis Transmission - 7 PLADs, China, 2019 and 2021
}

Junyi He'; Hongqing Zhu'; Ziping Bao'; Lijuan Zhang'; Yinlong Li'; Ting Feng'; Suying Guo'; Wangping Deng'; Can Wang'; Hui Dang'; Tiewu Jia'; Chao Lyu'; Zhiqiang Qin'; Chunli Cao ${ }^{1, *}$; Jing Xu'; Shizhu Li'; Xiaonong Zhou

\section{Summary \\ What is already known about this topic? \\ Oncomelania hupensis (O. hupensis) and livestock are main infection sources of schistosomiasis. The schistosome infected $O$. hupensis and livestock's feces are important risk factors in the transmission of schistosomiasis. \\ What is added by this report? \\ The potential risks of schistosomiasis transmission remain prevalent, giving an early warning to local government with information on existing transmission risks. It is expected that the effectiveness and efficiency of schistosomiasis surveillance could be improved by conducting rapid risk assessment at the beginning of transmission season. \\ What are the implications for public health practice? \\ Rapid risk assessment is essential in early detection and the active monitoring of indicators of the transmission risks of schistosomiasis in endemic areas. This could work synergistically with surveillance system to minimize infections and prevent rebounds of endemic schistosomiasis outbreaks.}

Schistosomiasis was endemic to 12 provincial-level administrative divisions (PLADs) in the middle and lower reaches of the Yangtze River and southern China. Oncomelania hupensis (O. hupensis) was the only intermediate snail host of Schistosoma japonicum (1). Previous studies had illustrated that the contribution of cattle feces to the transmission of schistosomiasis had accounted for $75 \%$ (2). In order to detect these potential risks of schistosomiasis transmission, indicators including distribution and infection rates of $O$. hupensis and livestock's feces around $O$. hupensis habitats were assessed at the beginning of transmission season in the 7 PLADs where schistosomiasis elimination had not achieved the national criteria (3). The rapid assessments were conducted in the spring of 2019 and 2021. The
O. hupensis survey was performed by means of environmental sampling, and livestock feces samples were collected in surveyed $O$. hupensis habitats. Loopmediated isothermal amplification (LAMP) assay and miracidial hatching were used to detect for $O$. hupensis infection and livestock's feces samples, respectively. In 2021, 2 surveyed environments detected nucleic acids of schistosomes among $O$. hupensis snails by LAMP assay. Schistosome eggs were found in cattle feces collected from snail habitats both in 2019 and 2021. These results indicated that the distribution and infections of $O$. hupensis and livestock's feces may cause concerns in endemic areas, which also gave an early warning that rapid response measures should be implemented in key environments to eliminate the potential risks of schistosomiasis transmission as soon as possible.

The assessments were conducted in the schistosomiasis endemic areas of Hunan, Hubei, Jiangxi, Anhui, Jiangsu, Sichuan, and Yunnan Provinces. Stratified random sampling was used to select surveyed environments. In each surveyed environment, over 50 square frames of $0.1 \mathrm{~m}^{2}$ were used to capture all $O$. hupensis within the frame. Every 10 specimens of captured $O$. hupensis were pooled in 1 centrifuge tube (1 tube for less than $10 \mathrm{O}$. hupensis) for DNA extraction, and DNA extracted from 50 $O$. hupensis samples were pooled for LAMP assay to detect S. japonicum. Surveyed environments with LAMP-tested positive $O$. hupensis were categorized as a positive environment. Livestock's feces found in surveyed $O$. hupensis fields were all collected. Stool samples were categorized as positive if schistosome miracidia was detected by the hatching test ( 3 bottles for 1 sample). The proportion of frames with living $O$. hupensis to total surveyed frames, rate of positive $O$. hupensis habitats, and positive livestock-feces samples were calculated.

A total of 33 administrative villages in 14 counties of the 7 PLADs were sampled in 2019 and 2021 without 
duplicate sampled counties (Table 1-2). In 2019, 68 $O$. hupensis inhabited environments were surveyed, in which $41.18 \%$ (28/68) environments had O. hupensis captured. Out of the total of 3,115 surveyed frames, living $O$. hupensis occurred in 324 frames. A total of 1,919 O. hupensis were living of all 2,076 captured, and an irrigation ditch in Laoguanju Village (Hubei Province) had the highest number of living $O$. hupensis in single frame at 69/frame. The LAMP assay detected no positive environments from all surveyed fields. A total of 56 stool samples were found in 7 sampled counties' surveyed environments, including 53 $(94.64 \%)$ cattle-feces samples and $3(5.36 \%)$ sheepfeces samples. A total of 4 cattle-feces sample from a marshland in Minglang Village (Hunan Province) tested positive by miracidial hatching, with a $7.14 \%$ (4/56) positivity rate of livestock-feces samples (Table 1).

In 2021, 74 O. hupensis inhabited environments were surveyed, in which $50 \%$ (37/74) of environments had $O$. hupensis captured. Of the total of 3,532 surveyed frames, living O. hupensis occurred in 292 frames. In addition, of all 1,958 O. hupensis captured,
1,870 were living. In marshland and lake endemic areas, an irrigation ditch in Lianyi Village (Hubei Province) had the highest number of living $O$. hupensis in a single frame at $94 /$ frame. In mountainous and hilly endemic areas, a tobacco field in Yunfeng Village (Yunnan Province) had the highest number of living O. hupensis in a single frame at $31 /$ frame. The LAMP assay detected 2 positive environments where the nucleic acids of a schistosome were found in $O$. hupensis, including a marshland in Wufeng Village (Jiangxi Province) and an irrigation ditch in Lianyi Village (Hubei Province), with a 2.70\% (2/74) positivity rate of $O$. hupensis habitats. A total of 54 stool samples were found in 13 surveyed environments in 9 sampled counties, including cattle feces, sheep feces, and other mammal's feces, accounting for $42.59 \%(23 / 54), \quad 38.89 \% \quad(21 / 54)$, and $18.52 \%$ (10/54), respectively. One cattle-feces sample from a marshland in Lianxu Village (Jiangxi Province) tested positive by miracidial hatching, with a $1.85 \%(1 / 54)$ positivity rate of livestock-feces samples (Table 2).

TABLE 1. Results of rapid assessments on potential risks of schistosomiasis transmission in 7 PLADs, China, Spring 2019.

\begin{tabular}{|c|c|c|c|c|c|c|c|}
\hline \multirow[b]{2}{*}{ Province } & \multirow[b]{2}{*}{$\begin{array}{l}\text { County/City/ } \\
\text { District }\end{array}$} & \multirow[b]{2}{*}{$\begin{array}{l}\text { Number of } \\
\text { investigated } \\
\text { villages }\end{array}$} & \multirow[b]{2}{*}{$\begin{array}{c}\text { Number of } \\
\text { surveyed } \\
\text { environments }\end{array}$} & \multicolumn{2}{|c|}{ O. hupensis survey result } & \multicolumn{2}{|c|}{ Livestock's feces survey result } \\
\hline & & & & $\begin{array}{c}\text { Frames with living } 0 . \\
\text { hupensis/total surveyed } \\
\text { frames }\end{array}$ & $\begin{array}{l}\text { LAMP } \\
\text { result* }\end{array}$ & Count & $\begin{array}{l}\text { Miracidial hatching } \\
\text { positive }\end{array}$ \\
\hline \multirow{2}{*}{ Jiangsu } & Jiangning District & 2 & 7 & $52 / 204$ & 0 & 0 & 0 \\
\hline & Pukou District & 4 & 6 & $39 / 187$ & 0 & 0 & 0 \\
\hline \multirow{2}{*}{ Anhui } & Wuwei County & 2 & 4 & $3 / 210$ & 0 & 8 & 0 \\
\hline & Sanshan District & 2 & 3 & $0 / 160$ & 0 & 0 & 0 \\
\hline \multirow{2}{*}{ Jiangxi } & Pengze County & 2 & 4 & $13 / 222$ & 0 & 0 & 0 \\
\hline & Ruichang City & 3 & 5 & $30 / 271$ & 0 & 0 & 0 \\
\hline \multirow{2}{*}{ Hubei } & Chibi City & 3 & 6 & $30 / 303$ & 0 & 3 & 0 \\
\hline & Jiayu County & 3 & 6 & $20 / 306$ & 0 & 0 & 0 \\
\hline \multirow{2}{*}{ Hunan } & Ziyang District & 2 & 4 & $81 / 205$ & 0 & 18 & 4 \\
\hline & Nan County & 2 & 4 & $32 / 203$ & 0 & 20 & 0 \\
\hline \multirow{2}{*}{ Sichuan } & Dechang County & 2 & 5 & $2 / 205$ & 0 & 0 & 0 \\
\hline & Xichang City & 2 & 6 & $19 / 239$ & 0 & 3 & 0 \\
\hline \multirow{2}{*}{ Yunnan } & Yongsheng County & 2 & 4 & $3 / 200$ & 0 & 2 & 0 \\
\hline & Jianchuan County & 2 & 4 & $0 / 200$ & 0 & 2 & 0 \\
\hline Total & & 33 & 68 & $324 / 3,115$ & 0 & 56 & 4 \\
\hline
\end{tabular}

Note: Based on national schistosomiasis epidemic data in the last 2 years, 2 epidemic counties (cities, districts) with relatively intense history of epidemics were selected from each province, 2 towns were selected from each sampled county (city, district), 1 village with intense history of epidemics and large extant areas of $O$. hupensis habitats was selected from each sampled town, and 2 possible 0. hupensis inhabited environments were selected from each sampled village to conduct the field survey.

Abbreviations: PLADs=provincial-level administrative divisions; LAMP=loop-mediated isothermal amplification; O. hupensis=Oncomelania hupensis.

* LAMP results: 1: Positive, 0: Negative. 
TABLE 2. Results of rapid assessments on potential risks of schistosomiasis transmission in 7 PLADs, China, Spring 2021.

\begin{tabular}{|c|c|c|c|c|c|c|c|}
\hline \multirow[b]{2}{*}{ Province } & \multirow[b]{2}{*}{$\begin{array}{c}\text { County/City/ } \\
\text { District }\end{array}$} & \multirow{2}{*}{$\begin{array}{c}\text { Number of } \\
\text { investigated } \\
\text { villages }\end{array}$} & \multirow{2}{*}{$\begin{array}{c}\text { Number of } \\
\text { surveyed } \\
\text { environments }\end{array}$} & \multicolumn{2}{|c|}{ O. hupensis survey result } & \multicolumn{2}{|c|}{ Livestock's feces survey result } \\
\hline & & & & $\begin{array}{l}\text { Frames with living } \\
0 . \text { hupensis/total } \\
\text { surveyed frames }\end{array}$ & $\begin{array}{l}\text { LAMP } \\
\text { result* }\end{array}$ & Count & $\begin{array}{l}\text { Miracidial hatching } \\
\text { positive }\end{array}$ \\
\hline \multirow{2}{*}{ Jiangsu } & New Zhenjiang District & 2 & 4 & $2 / 213$ & 0 & 1 & 0 \\
\hline & Yangzhong City & 2 & 4 & $3 / 208$ & 0 & 3 & 0 \\
\hline \multirow{2}{*}{ Anhui } & Wanzhi Distrcit & 2 & 6 & $31 / 223$ & 0 & 21 & 0 \\
\hline & Xuanzhou District & 2 & 9 & $14 / 219$ & 0 & 0 & 0 \\
\hline \multirow{2}{*}{ Jiangxi } & Jinxian County & 2 & 3 & $0 / 162$ & 0 & 4 & 0 \\
\hline & Nanchang County & 3 & 5 & $11 / 293$ & 1 & 13 & 1 \\
\hline \multirow{2}{*}{ Hubei } & Shashi District & 3 & 8 & $1 / 439$ & 0 & 1 & 0 \\
\hline & Jingzhou District & 3 & 6 & $42 / 187$ & 1 & 2 & 0 \\
\hline \multirow{2}{*}{ Hunan } & Hanshou District & 2 & 4 & $8 / 209$ & 0 & 0 & 0 \\
\hline & Dincheng District & 3 & 4 & $18 / 209$ & 0 & 6 & 0 \\
\hline \multirow{2}{*}{ Sichuan } & Mianzhu City & 2 & 4 & $88 / 240$ & 0 & 0 & 0 \\
\hline & Jingyang District & 2 & 4 & $63 / 233$ & 0 & 0 & 0 \\
\hline \multirow{2}{*}{ Yunnan } & Heqing County & 3 & 8 & $0 / 435$ & 0 & 3 & 0 \\
\hline & Dali City & 2 & 5 & $11 / 262$ & 0 & 0 & 0 \\
\hline Total & & 33 & 74 & $292 / 3,532$ & 2 & 54 & 1 \\
\hline
\end{tabular}

Note: Based on national schistosomiasis epidemic data in the last 2 years, 2 epidemic counties (cities, districts) with relatively intense history of epidemics were selected from each province, 2 towns were selected from each sampled county (city, district), 1 village with intense history of epidemics and large extant areas of $O$. hupensis habitats was selected from each sampled town, and 2 possible 0. hupensis inhabited environments were selected from each sampled village to conduct the field survey.

* LAMP results: 1: Positive, 0: Negative.

Abbreviations: PLADs=provincial-level administrative divisions; LAMP=loop-mediated isothermal amplification; O. hupensis=Oncomelania hupensis.

\section{DISCUSSION}

Even though the current prevalence of schistosomiasis was relatively low across China (3), rapid assessments in 2019 and 2021 have found that the density of $O$. hupensis distribution remained high in several $O$. hupensis habitats and surrounding environments where fecal contamination was also observed. In addition, the infections of $O$. hupensis and livestock's feces were both detected in the assessments. The potential risks of schistosomiasis transmission are likely remaining prevalent, which is an early warning of the transmission risks of schistosomiasis at the beginning of transmission season. This finding further indicated that low sensitivity existed in schistosomiasis surveillance system. By conducting rapid risk assessments before the beginning of transmission season, the effectiveness and efficiency of schistosomiasis surveillance may be improved.

In the index system for rapid assessment of environments with high transmission risk of schistosomiasis, morbidity in livestock and $O$. hupensis were regarded as the primary indices, and the secondary indices included the infection rate of livestock, areas with infected $O$. hupensis, and density of infected $O$. hupensis (4). This index system showed the importance of assessing transmission risks related to $O$. hupensis and livestock in not only building a comprehensive risk assessment system but also in realizing earlier detection and response to further reduce the possible infection in $O$. hupensis and livestock. Thus, the distribution and infection of $O$. hupensis and livestock's feces were assessed as essential indicators in the rapid assessments and surveillance on schistosomiasis transmission.

The existence and reemergence of $O$. hupensis can greatly affect the transmission of schistosomiasis across endemic areas in 12 southern PLADs (1). The optimal temperature for living O. hupensis is $20-25{ }^{\circ} \mathrm{C}$, and their main multiplying stage is between April and June (5), during which people and livestock may be more susceptible to schistosomiasis infections. Especially in marshland and lake endemic areas, the distribution of $O$. hupensis and the transmission of schistosomiasis may be facilitated after encountering flood disasters (6). In the risk assessment of 5 PLADs affected by 
flood disasters in the summer of 2020, 3,240 living $O$. hupensis were captured out of all 8,904 captured, and 1 LAMP-tested positive $O$. hupensis-inhabited environment was detected in the 64 assessed environments (7). The flood also caused $O$. hupensis to reemerge in a few environments where no $O$. hupensis distribution had been reported over the past 20 years at least, extending the distribution of $O$. hupensis ( 7 ).

Livestock is another key factor that causes schistosomiasis transmission. It is reported that cattle feces infected with the schistosome eggs can account for $80 \%$ to $90 \%$ of schistosomiasis transmission (8). Even though several measures have been taken to prevent schistosomiasis transmission caused by livestock activities, such as replacing farming cattle with machines, prohibiting grazing on $O$. hupensisinfested grassland and marshland (2), a few livestock, including cattle and sheep, were found grazing around $O$. hupensis habitats during the assessments. The reemergence of livestock activities may lead to a rebound of schistosomiasis infections in endemic areas (9). In terms of positive feces detected, harmless disposal of feces was conducted to prevent the transmission. The management of livestock activities should be strengthened as well.

This study was subjected to some limitations. First, the assessment was not conducted in 2020 due to the coronavirus disease 2019 (COVID-19) pandemic, which affected the continuity of assessments. There was 1 case of acute schistosomiasis reported in 2020 (3); therefore, assessing environments with transmission risks of schistosomiasis annually is needed based on historical reports and data to avoid infections (10). Second, only environmental sampling was applied in investigating $O$. hupensis habitats, which was not as comprehensive as systematic sampling applied in regular risk surveillance. Although our findings can point out potential risks by reporting the distribution and infection of these indicators in endemic regions, further assessment and surveillance should be performed on a broader scale.

In conclusion, in order to achieve the goal of eliminating schistosomiasis in China before 2030 (2), environments with potential risks of schistosomiasis transmission need prompt and efficient detection and surveillance to minimize the possibility of schistosomiasis infection in both humans and livestock. Rapid risk assessment should also be applied to actively monitor indicators of the transmission risks of schistosomiasis and prevent the rebound of the endemic schistosomiasis outbreaks.

Acknowledgements: Participants of the survey.

Conflicts of interest: No conflicts of interest declared.

doi: $10.46234 / \mathrm{ccdcw} 2021.263$

\# Corresponding author: Chunli Cao, caocl@nipd.chinacdc.cn.

\begin{abstract}
${ }^{1}$ National Institute of Parasitic Diseases, Chinese Center for Disease Control and Prevention (Chinese Center for Tropical Diseases Research), NHC Key Laboratory of Parasite and Vector Biology, WHO Collaborating Centre for Tropical Diseases, National Center for International Research on Tropical Diseases, Shanghai, China.
\end{abstract}

Submitted: September 23, 2021; Accepted: November 26, 2021

\section{REFERENCES}

1. Li ZJ, Ge J, Dai JR, Wen LY, Lin DD, Madsen H, et al. Biology and control of snail intermediate host of Schistosoma japonicum in the People's Republic of China. Adv Parasitol 2016;92:197 - 236. http:// dx.doi.org/10.1016/bs.apar.2016.02.003.

2. Lv S, Lv C, Li YL, Xu J, Hong QB, Zhou J, et al. Expert consensus on the strategy and measures to interrupt the transmission of schistosomiasis in China. Chin J Schisto Control 2021;33(1):10 - 4. http://dx.doi.org/10.16250/j.32.1374.2021007. (In Chinese).

3. Zhang LJ, Xu ZM, Yang F, Dang H, Li YL, Lv S, et al. Endemic status of schistosomiasis in People's Republic of China in 2020. Chin J Schisto Control 2021;33(3):225 - 33. http://dx.doi.org/10.16250/j.32. 1374.2021109. (In Chinese).

4. Cao CL, Xu JF, Xu J, Bao ZP, Jia TW, Yu Q, et al. Establishment and application of rapid assessment system of environment with high transmission risk of schistosomiasis in marshland and lake regions I Establishment of an index system with Delphi method. Chin J Schisto Control 2013;25(3):232 - 6. http://dx.doi.org/10.3969/j.issn.10056661.2013.03.004. (In Chinese).

5. Zhou XN. Science on Oncomelania snail. Beijing: Science Press. 2005; p.77,149.http://find.nlc.cn/search/showDocDetails?docId=1072718675 $644676334 \&$ dataSource $=$ ucs $01 \&$ query $=\%$ E $\% 92 \% 89 \%$ E $8 \% 9 \mathrm{E} \%$ BA $\%$ E7\%A7\%91\%E5\%AD\%A6. (In Chinese).

6. Zhang LJ, Zhu HQ, Wang Q, Lv S, Xu J, Li SZ. Assessment of schistosomiasis transmission risk along the Yangtze River basin after the flood disaster in 2020. Chin J Schisto Control 2020;32(5):464 - 8,475. http://dx.doi.org/10.16250/j.32.1374.2020242. (In Chinese).

7. Guo SY, Zhu HQ, Cao CL, Deng WP, Bao ZP, Jia TW, et al. Risk assessment of schistosomiasis transmission along the middle and lower Yangtze River after flooding in 2020. Chin J Parasitol Parasit Dis 2021;39(6):in press

8. Wang LD. The history and prospects of schistosomiasis control in China-An anthology for the 100th anniversary of the discovery of schistosomiasis in China. Beijing: People's Medical Publishing House. 2006; p. 234. (In Chinese).

9. Lin JJ. Endemic status and control of animal schistosomiasis in China. Chin J Schisto Control 2019;31(1):40 - 6. http://dx.doi.org/10.16250/ j.32.1374.2018313. (In Chinese).

10. Cao CL, Zhang LJ, Bao ZP, Dai SM, Lv S, Xu J, et al. Endemic situation of schistosomiasis in People's Republic of China from 2010 to 2017. Chin J Schisto Control 2019;31(5):519 - 21,554. http://dx.doi. org/10.16250/j.32.1374.2018232. (In Chinese). 